\title{
Human papillomavirus E6-regulated microRNA-20b promotes invasion in cervical cancer by targeting tissue inhibitor of metalloproteinase 2
}

\author{
YUAN CHENG $^{1}$, LI GENG $^{2}$, LIJUN ZHAO $^{1}$, PENG ZUO $^{1}$ and JIANLIU WANG ${ }^{1}$ \\ ${ }^{1}$ Department of Gynecology and Obstetrics, Peking University People's Hospital, Beijing 100044; \\ ${ }^{2}$ Department of Gynecology and Obstetrics, Peking University Third Hospital, Beijing 100191, P.R. China
}

Received March 14, 2017; Accepted August 3, 2017

DOI: $10.3892 / \mathrm{mmr} .2017 .7231$

\begin{abstract}
Human papillomavirus (HPV) infection alone is not sufficient for development of cervical cancer and further risk factors are involved, however, the underlying mechanism remains to be elucidated. The authors previously used a microarray assay to reveal microR-20b (miR-20b) as a key node in the miRNA-mRNA network of cervical carcinoma. The present study demonstrated an increased expression of miR-20b in cervical carcinoma tissue. MiR-20b was regulated by HPV E6 oncoprotein in cervical cancer. Furthermore, miR-20b overexpression with mimics induced cell morphological alterations and the epithelial-mesenchymal transition. Treating cervical cancer cells with the miR-20b inhibitor decreased the migration and invasion of cervical cancer cells. Tissue inhibitor of metalloproteinase 2 (TIMP-2), a possible antagonist of matrix metalloproteinase 2, is a metastasis suppressor and predicted to be a potential target of miR-20b. Fluorescence signals were decreased on transducing HeLa cells with a TIMP-2 3'-untranslated region plasmid and miR-20b mimics compared with control. Finally, TIMP-2 was identified as a novel target of miR-20b and was demonstrated to be regulated by the HPV oncoprotein. In addition, miR-20b and TIMP-2 were involved in cell invasion regulated by HPV E6. The present study demonstrated a novel pathway of HPV/miR-20b/TIMP-2 during the process of invasion in cervical cancer cells.
\end{abstract}

Correspondence to: Professor Jianliu Wang, Department of Gynecology and Obstetrics, Peking University People's Hospital, 11 Xizhimen South Street, Beijing 100044, P.R. China

E-mail: wangjianliu@pkuph.edu.cn

Professor Li Geng, Department of Gynecology and Obstetrics, Peking University Third Hospital, 49 Huayuan-Bei Road, Beijing 100191, P.R. China

E-mail: gengli1957@bjmu.edu.cn

Key words: miR-20b, HPV, cervical cancer, TIMP-2, migration, invasion

\section{Introduction}

Cervical cancer is a frequent gynecological malignant tumor that threatens the life and health of many women (1). Persistent infection with human papillomavirus (HPV) is one of the greatest risk factors for cervical cancer (2). However, HPV infection alone is not sufficient for development of cervical cancer; some other risk factors are involved (3). Nevertheless, some of the underlying regulatory mechanisms are not completely understood.

MicroRNAs (miRs) are a class of small-molecule RNAs of 21 to $24 \mathrm{nt}$ that participate in various stages of the occurrence of tumor by negatively regulating downstream target mRNAs or protein (4). MiRNAs in cervical cancer are still being identified. MiRNAs participate in the genesis and progression of cervical cancer and their expression is strongly associated with that of HPV oncoproteins (5). Our previous microarray assay revealed miR-20b as a key node in the miRNA-mRNA network of cervical carcinoma (6). MiRNA-20b is a member of the miRNA 106a-363 cluster, which belongs to miR-17 family. Members of miR17 family are frequently involved in many human cancers. The role of miR-20b in cancer has been recently revealed. Leidinger et al confirmed that miR-20b can be used as a blood marker to accurately distinguish between non-small cell lung cancer and controls (7). Aberrant expression of miR-20b was found related to a chemotherapeutic response induced by hypoxia in gastric cancer (8). Anti-angiomiR miR-20b was used as potential therapeutic target for refractory large B-cell lymphoma (9). However, the role of miR-20b in cancer of the cervix has not been explored.

In this study, we investigated miR-20b expression in cervical cancer tissue and cells. MiR-20b promoted cancer migration and invasion and the epithelial-mesenchymal transition (EMT), a phenotypic switch whereby epithelial cells acquire mesenchymal attributes, a critical step attributed to metastases (10). Tissue inhibitor of metalloproteinase 2 (TIMP-2) is predicted to be a potential target of miR-20b by TargetScan software. TIMP-2 is a unique endogenous inhibitor for extracellular MMPs. MMPs produced by tumor cells or tumor-associated fibroblasts play an important role in cancer metastasis. MMPs and TIMP-2 have an important role in cervical cancer progression. In our study, we found tissue inhibitor of metalloproteinase 2 (TIMP-2) as a 
direct target of miR-20b in cervical cancer and the regulation of both by HPV E6. Moreover, miR-20b and TIMP-2 were involved in invasion regulated by HPV18 E6. Finally, we demonstrated a novel pathway of HPV/miR-20b/TIMP-2 during the process of invasion in cervical cancer cells.

\section{Materials and methods}

Human samples and cell lines. Cervical tissues were acquired from patients in the Department of Obstetrics and Gynecology, Peking University Third Hospital (Beijing, China). The study was approved by the Ethics Committee of Peking University Third Hospital (Ethical lot no. 2014025). Informed consent was obtained from all patients. Specimens were obtained within 5 min after surgery with liquid nitrogen transport, then stored at $-80^{\circ} \mathrm{C}$ for extraction of RNA. C33A (HPV-), HeLa (HPV18+), SiHa and Caski (HPV16+) human cervical cancer cell lines were kept in the laboratory of Peking University People's Hospital. SiHa and HeLa cells were cultured in DMEM medium. C33A and CaSki cells were cultured in MEM and RPMI1640 medium, respectively, with $10 \%$ fetal bovine serum (FBS) at $37^{\circ} \mathrm{C}$ and $5 \% \mathrm{CO}_{2}$.

Treatment and reagents. Cells were transfected with miRNA mimics and inhibitors, pmir-GLO plasmid, HPV E6 siRNA or TIMP-2 siRNA, by means of Lipofectamine 2000 (Invitrogen, Carlsbad, CA, USA). The primer sequence for miR-20b was 5'-CAAAGTGCTCATGTGCAGGTAG-3'. We used mature miR-20b mimics and inhibitors (Ribobio, Guangzhou, China), HPV18 E6 siRNA and TIMP-2 siRNA (GenePharma, Shanghai, China), and antibodies for anti-HPV18E6 (Abcam, Cambridge, USA), E-cadherin, N-cadhein, vimentin, TIMP-2 (Cell Signaling Technology), and GAPDH (Santa Cruz Biotechnology, Inc., Santa Cruz, CA).

Cell migration assays. Cells were cultured in serum-free medium in a single layer and covered with a dish for scratch assay. A $20-\mu 1$ pipette tip was used to create one vertical wound in each well, which was photographed by inverted microscopy at 0 and $24 \mathrm{~h}$. The healing area was calculated.

Cell invasion assays. The membrane of the Transwell chamber (Costar) was pre-coated with $50 \mathrm{mg} / 1$ Matrigel solution. Cells cultured in serum-free medium were seeded in the upper chamber. The lower chamber contained DMEM with $10 \%$ FBS. After $24 \mathrm{~h}$, Transwell chambers were rinsed with $1 \%$ phosphate buffered saline (PBS). Cells on the upper surface were removed. The whole chamber was fixed in 4\% paraformaldehyde for $15 \mathrm{~min}$ and dried, then stained with crystal violet for $10 \mathrm{~min}$. The number of migrating cells was counted under an inverted microscope.

Luciferase reporter assay. Wild-type or mutant human TIMP-2 mRNA 3'UTR were cloned into pmir-GLO plasmids with the dual luciferase reporter gene. The plasmids were verified by sequencing. HeLa cells were seeded into 6-well plates and co-transfected with miR-20b mimics or negative control and 3'UTR vectors. Luciferase assays involved use of the Luciferase Reporter Gene Assay kit (Promega) and activity was normalized to Renilla luciferase activity.
Statistical analysis. Data are described as mean \pm SEM, and GraphPad Prism 5 was used for analysis. Data were compared by Student's t test or one- or two-way ANOVA. P<0.05 was considered to indicate a statistically significant difference.

\section{Results}

High expression of miR-20b regulated by HPV E6 in cervical cancer. Our previous miRNA microarray assay showed miR-20b upregulated in cervical cancer and a key node in the miRNA-gene network (6). To validate our microarray results, we further evaluated the expression of miR-20b in 19 cervical carcinoma samples and 19 normal cervical samples. MiR-20b expression was high in human cervical carcinoma samples (Fig. 1A).

A variety of cervical cancer cell lines have been established from different types of cervical cancer patients. HeLa is human papilloma virus 18 type cell line from cervical adenocarcinoma. CaSki and $\mathrm{SiHa}$ are human papilloma virus 16 type cervical squamous cancer cell lines (11). We examined the expression of miR-20b in 4 cervical cancer cell lines. MiR-20b was upregulated in HPV-positive cervical cancer cell lines (Hela HPV18+, Caski and SiHa HPV16+ cells), whatever the pathological type, as compared with C33A cells, which do not carry HPV (Fig. 1B). Next, we inhibited HPV E6 by using siRNA and found E6 mRNA level markedly suppressed with siRNA treatment (Fig. 1C). We also observed that the expression of miR-20b decreased after inhibition of HPVE6 (Fig. 1D).

MiR-20b induced morphologic changes in HeLa cells and promoted their migration and invasion. We further investigated the effect of miR-20b in cervical carcinoma. First, we used mimics and inhibitors to knock down and overexpress miR-20b respectively (Fig. 2A). MiR-20b could induce cells to slightly adopt a long shuttle type (Fig. 2B). We examined whether miR-20b affected cell migration and invasion. Cell scratch assay revealed that with $\mathrm{miR}-20 \mathrm{~b}$ mimic overexpression, cells significantly migrated to cover the wounded area, which was decreased with miR-20b inhibition (Fig. 2C and D). Inhibiting miR-20b decreased the invasion of HeLa cells across the chamber membrane, and miR-20b mimic overexpression promoted the invasion (Fig. 2E and F).

miR-20b enhanced EMT. The EMT process is often accompanied by cell morphological changes. E-cadherin is expressed in normal epithelial cells. The loss of E-cadherin results in reduced cell adhesion ability and cytoskeletal rearrangement. Cancer cells feature a low expression of E-cadherin. $\mathrm{N}$-cadherin and vimentin, EMT markers, are upregulated in various cancer cells. Our results showed that overexpression of miR-20b led to decreased E-cadherin protein level but increased $\mathrm{N}$-cadherin and vimentin levels as compared with the vector control (Fig. 3A-C). Consistent with this result, miR-20b inhibitor increased the protein expression of E-cadherin and suppressed that of $\mathrm{N}$-cadherin and vimentin (Fig. 3D-F).

HPV E6 and miR-20b regulated the protein expression of TIMP-2, and miR-20b targeted TIMP-2. The mechanism of miRNA positive regulation on cancer cells was revealed 
A

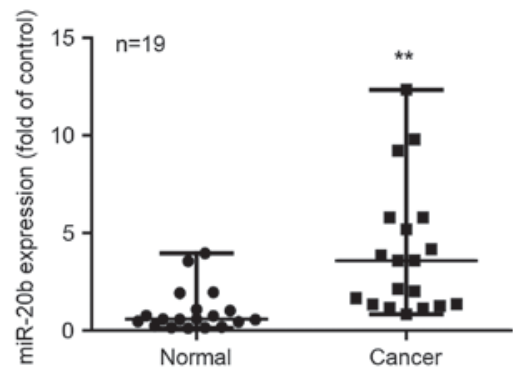

C

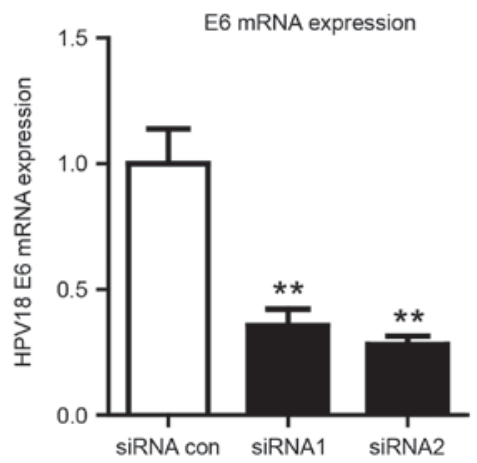

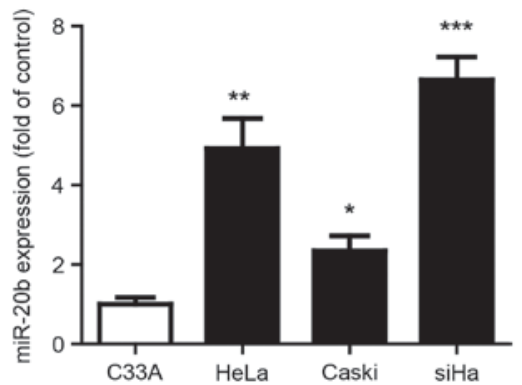

D

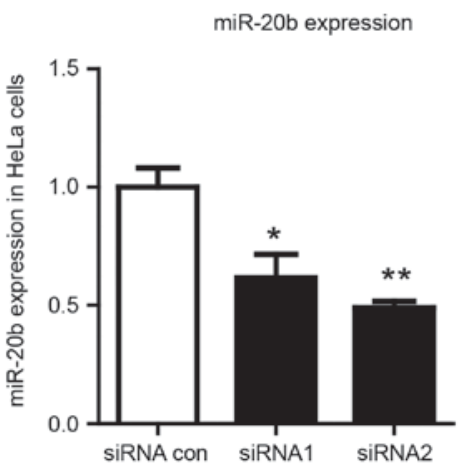

Figure 1. High expression of miR-20b regulated by human papillomavirus (HPV) E6 in cervical cancer. (A) qPCR analysis of mRNA expression of miR-20b in human cervical tissues. U6 small nuclear RNA was an internal control. Each dot represents 1 sample. Horizontal line is mean, whiskers are SEM. n=19, ${ }^{* *} \mathrm{P}<0.01$ vs. vehicle. (B) RT-PCR analysis of the mRNA expression of miR-20b in human cervical cancer cell lines. C33A cells (HPV-) were a control. Data are mean \pm SEM $(n=3) . ~ P<0.05,{ }^{* * *} \mathrm{P}<0.01,{ }^{* * * *} \mathrm{P}<0.001$ vs. control. RT-PCR analysis of mRNA level of (C) HPV18 E6 and (D) miR-20b in HeLa cells after knockdown of HPV18E6 with 2 siRNAs." $\mathrm{P}<0.05 ;{ }^{* *} \mathrm{P}<0.01$.

via base-paring with target genes to repress the expression. TIMP-2 is a predicted target of miR-20b by Targetscan software (Fig. 4A). TIMP-2 is a unique endogenous inhibitor for extracellular MMPs. MMPs produced by tumor cells or tumor-associated fibroblasts play an important role in cancer metastasis. To determine whether TIMP-2 is downregulated by miR-20b, we first examined the mRNA expression of TIMP-2 in response to miR-20b. MiR-20b had no effect on TIMP-2 mRNA expression (Fig. 4B). Importantly, TIMP-2 protein level was indeed reduced with overexpression of miR-20b in HeLa cells as compared with controls (Fig. 4C). Furthermore, TIMP-2 was found a direct target of miR-20b, as seen on luciferase assay: miR-20b inhibited TIMP-2 luciferase activity on transfection of the wild-type plasmid (TIMP-2-WT-3'UTR) (Fig. 4D).

In Fig. 1D, we observed that miR-20b expression is regulated by HPV E6. Whether HPV has an effect on the expression of TIMP-2 is unknown. When HPV E6 was knocked down with 2 siRNA, TIMP-2 was markedly upregulated (Fig. 4E and F).

MiR-20b and TIMP-2 are involved in cell invasion regulated by HPV18 E6. Our results showed that miR-20b promotes cell invasion. TIMP-2 is known to be associated with cell invasion. So, we first inhibited miR-20b and knocked down TIMP-2 with siRNA to analyze the interaction between the two during cell invasion. The number of invading cells was increased with TIMP-2 knockdown after anti-miR-20b treatment (Fig. 5A). Previous results suggest that miR-20b can be upregulated by HPV E6, so we wondered whether miR-20b is involved in HPV E6-induced cell invasion. We found significantly increased invasion of cells with miR-20b overexpression and HPV E6
siRNA knockdown as compared with HPV E6 knockdown alone (Fig. 5B). In addition, the invasion of cells was increased with simultaneous TIMP-2 and HPV E6 siRNA knockdown as compared with HPV E6 knockdown alone (Fig. 5C).

\section{Discussion}

Our previous study with microarray assay found that miR-20b was a key node in the miR-mRNA network of cervical carcinoma (6). Here, we show that miR-20b is highly expressed in cervical carcinoma tissues. MiR-20b was upregulated in HPV-positive cervical cancer cell lines. The expression of miR-20b was downregulated with HPV E6 siRNA knockdown. This result suggests that the fate of miR-20b is closely related to HPV.

HPV infection plays an important role in abnormal miRNA expression. MiR-20b was found a potentially disease-free survival miRNA in HPV-associated oropharyngeal carcinoma (12). MiRNA array of HPV16 and HPV18 infection in organotypic raft cultures of foreskin and vaginal keratinocytes revealed that the viral oncoprotein $\mathrm{E} 6 / \mathrm{E} 7$ promotes the expression of miR-16, miR-25, miR-92a, and miR-378 (13). The authors confirmed that HPV regulates the expression of RNA in cervical cancer cells or the production of viral small RNA. As well, silencing E6/E7 in CaSki and SiHa cells affected the levels of miR-27b, miR-20a, miR-24, miR-93, and miR-106b. Finally, miR-27b regulated by E7 promoted cell proliferation and invasion of CaSki and SiHa cells (14). A series of studies have confirmed that HPV and miRNA have a close relationship and have become a new hot research area in cervical cancer. 
A

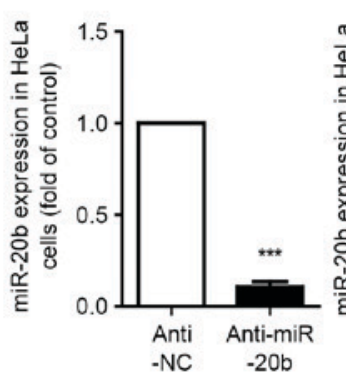

C
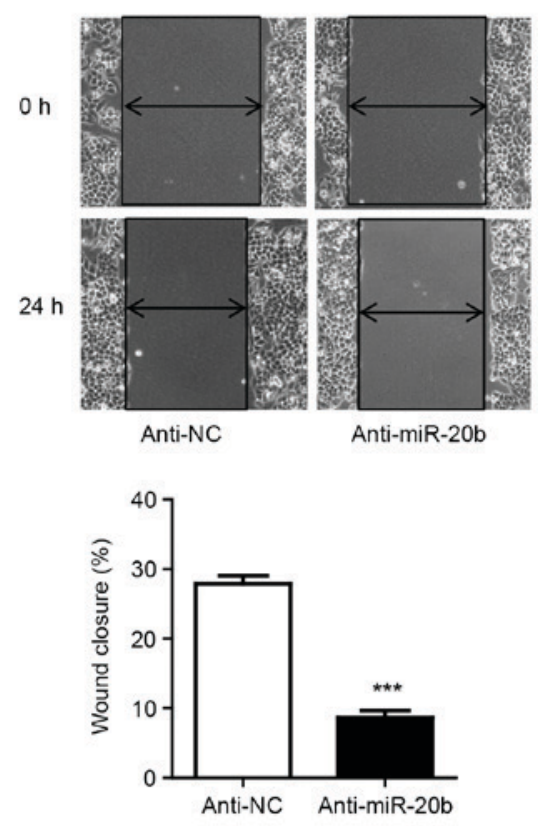

$\mathrm{E}$

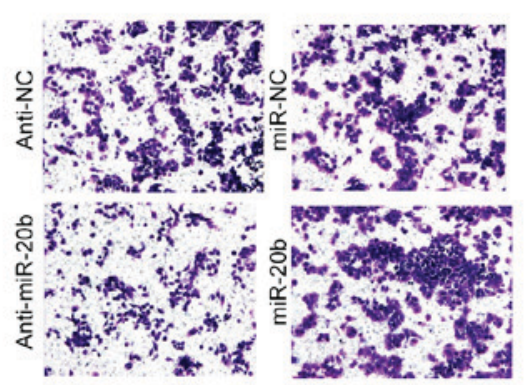

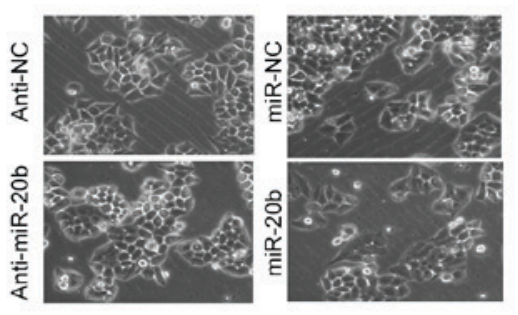

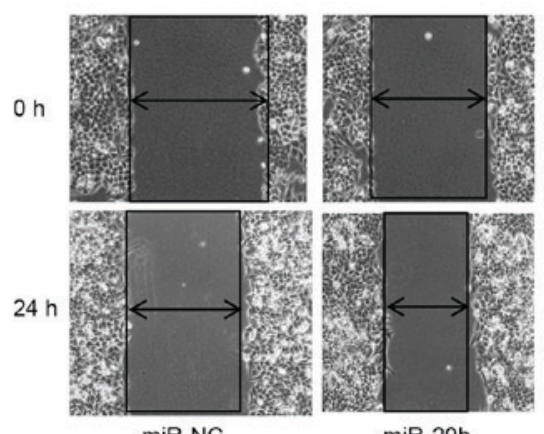

miR-NC

miR-20b

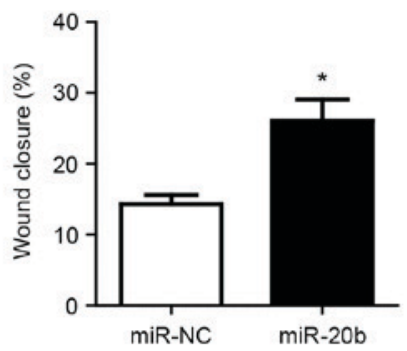

$\mathrm{F}$

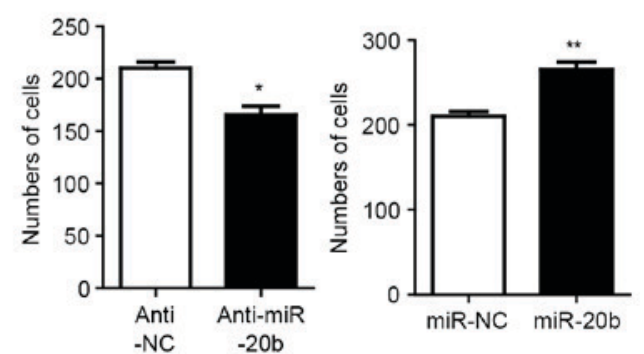

Figure 2. miR-20b induced morphologic changes of HeLa cells and promoted their migration and invasion. (A) Cells were transfected with miR-20b inhibitor (anti-miR-20b) at $100 \mathrm{~nm}$ and miR-20b mimics (miR-20b) at $50 \mathrm{~nm}$. Data are mean $\pm \mathrm{SEM}(\mathrm{n}=3)$. ${ }^{* *} \mathrm{P}<0.01,{ }^{* * * *} \mathrm{P}<0.001$ vs. control. (B) Microscopy of cell migration before and after miR-20b inhibition or overexpression. (C and D) Scratch assay of cell migration and (E and F) Transwell invasion assay of invasion with miR-20b inhibition and overexpression. Data are mean \pm SEM $(n=3) .{ }^{*} \mathrm{P}<0.05,{ }^{* *} \mathrm{P}<0.01,{ }^{* * * *} \mathrm{P}<0.001$ vs. control.

In recent years, the function of miR-20b in cancer has been gradually revealed. Our previous research revealed miR-20b as an indispensable network molecule in cervical cancer (6). In this study, we explored the important effect of miR-20b in cervical cancer. As compared with controls, miR-20b-overexpressing cells were more frequently spindle-shaped. MiR-20b may have some effects on the regulation of the cytoskeleton, EMT, migration and invasion. The EMT is a phenotypic switch whereby epithelial cells acquire mesenchymal attributes, a critical step attributed to metastases (10). EMT involves the remodeling of the cytoskeleton via a number of key genes, including decreased E-cadherin level and increased $\mathrm{N}$-cadherin and vimentin levels and many transcription factors $(15,16)$. Our data showed that the protein expression of EMT markers was regulated accordingly after gain or loss of miR-20b. Furthermore, our cell migration and invasion experiments confirmed the biological effects of miR-20b. Overexpression of miR-20b was an effective accelerator to promote migration and invasion of HeLa cells.

TIMP-2 is predicted to be a potential target of miR-20b by Targetscan software. TIMP-2 is a unique endogenous inhibitor for extracellular MMPs. MMPs produced by tumor cells or tumor-associated fibroblasts play an important role in cancer metastasis. MMPs/TIMP-2 have an important role in cervical cancer progression (17-19). Immunohistochemistry and mRNA in situ hybridization was used to detect the 
A
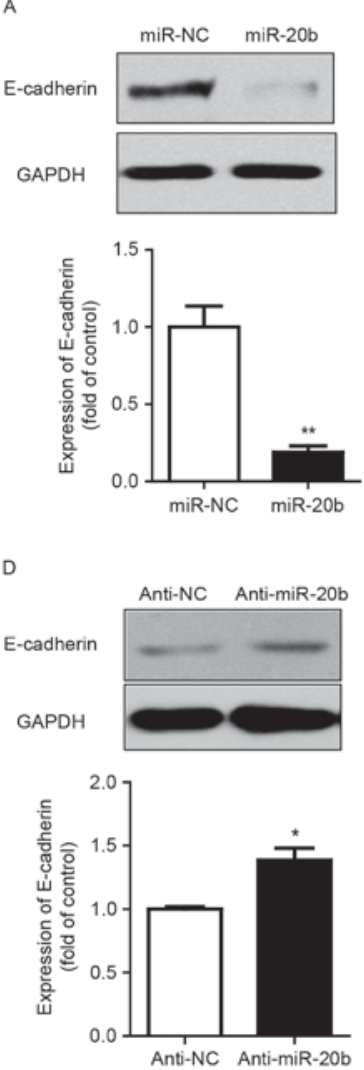
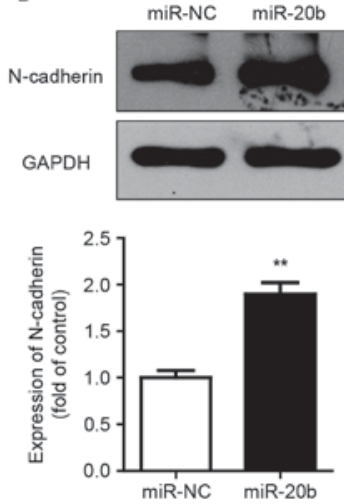

E
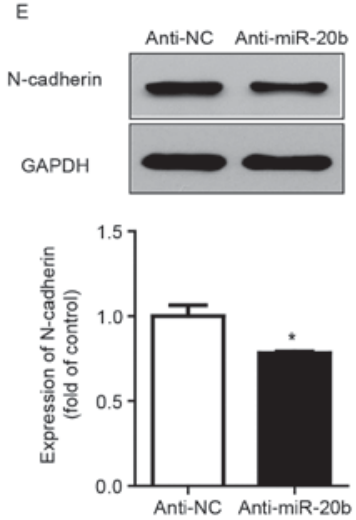
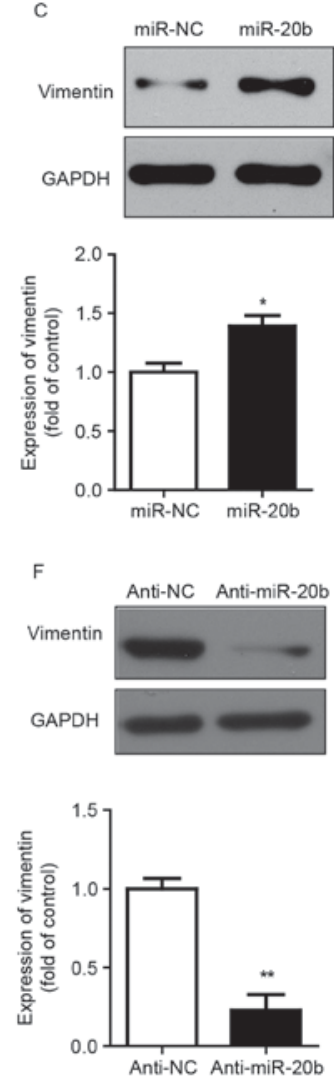

Figure 3. miR-20b enhanced EMT. Western blot analysis of protein levels of E-cadherin, N-cadherin and Vimentin after (A-C) overexpression and (D-F) inhibition of miR-20b in HeLa cells. GAPDH was an internal control. Data are mean \pm SEM $(n=3)$. ${ }^{*}<<0.05,{ }^{* *} \mathrm{P}<0.01$, compared to control.
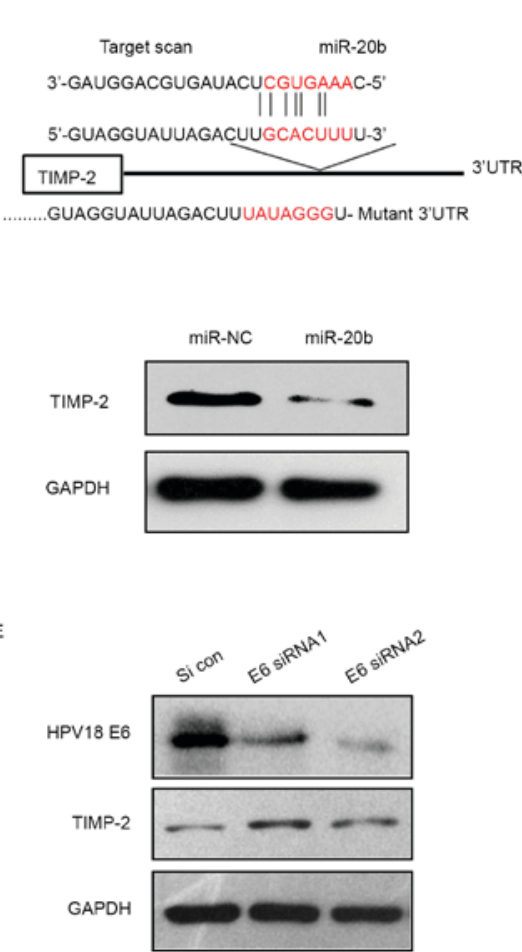
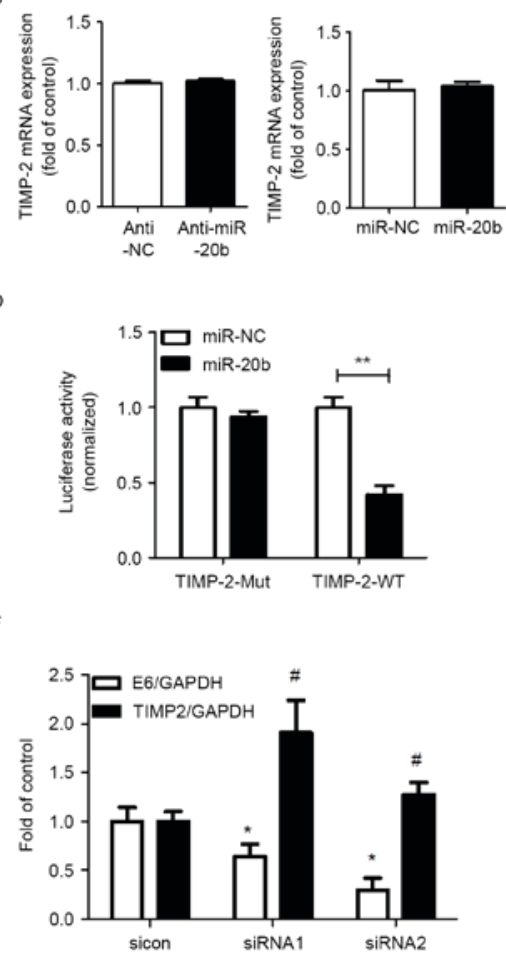

Figure 4. HPV and miR-20b regulated the expression of TIMP-2 protein, and miR-20b targeted it. (A) The predicted interaction site of miR-20b and candidate target gene TIMP-2 3'UTR. (B) qPCR analysis of TIMP-2 mRNA level in HeLa cells transfected with miR-106b inhibitor (anti-miR-106b) or miR-106b control inhibitor (anti-NC). Data are mean \pm SEM $(n=3)$. (C) Western blot analysis of TIMP-2 protein level in HeLa cells transfected with miR-106b inhibitor (anti-miR-106b) or miR-106b control inhibitor (anti-NC). (D) Luciferase assay of HeLa cells co-transfected with miR-20b mimics and pGL-3-TIMP-2 plasmid (miR-NC and miR-106b with TIMP-2-WT-3'UTR; miR-NC and miR-106b with TIMP-2-Mut-3'UTR) after $24 \mathrm{~h}$. Data are mean \pm SEM ( $\mathrm{n}=3$ ). ${ }^{* *} \mathrm{P}<0.01$. (E) TIMP-2 protein level after siRNA knockdown of HPV18 E6 protein and (F) quantification. Data are mean \pm SEM $(n=3)$. ${ }^{P} \mathrm{P}<0.05$ vs. control, ${ }^{*} \mathrm{P}<0.05$ vs. E6/GAPDH. 

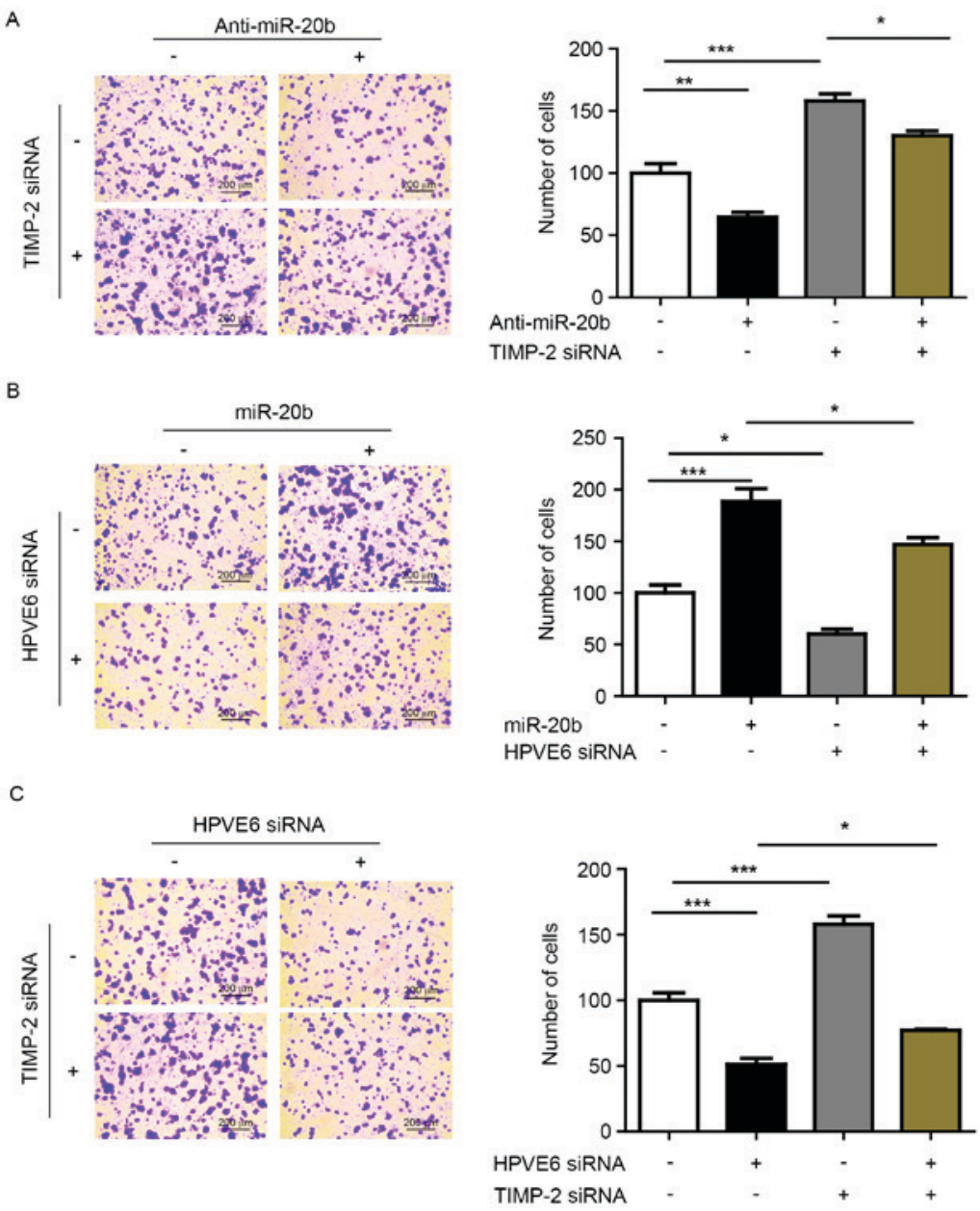

Figure 5. MiR-20b and TIMP-2 were involved in invasion regulated by HPV18E6. (A) HeLa cells were treated with or without anti-miR-20b and TIMP-2 siRNA and cell invasion was observed. ${ }^{*} \mathrm{P}<0.05,{ }^{* * *} \mathrm{P}<0.01,{ }^{* * * *} \mathrm{P}<0.001$. (B) HeLa cells were treated with or without miR-20b mimics and HPVE6 siRNA and cell invasion was observed. ${ }^{*} \mathrm{P}<0.05,{ }^{* * *} \mathrm{P}<0.001$. (C) HeLa cells were treated with or without TIMP-2 siRNA and HPVE6 siRNA and cell invasion was observed. ${ }^{*} \mathrm{P}<0.05,{ }^{* * * *} \mathrm{P}<0.001$.
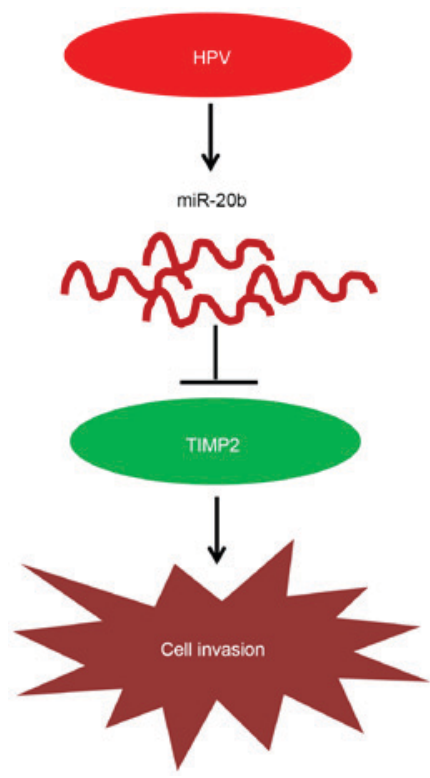

Figure 6. miR-20b regulated by HPVE6 promotes invasion in cervical cancer by targeting TIMP-2. Graphical abstract. MiR-20b regulated by HPVE6 promotes invasion in cervical cancer by targeting TIMP-2. The red oval represents the HPV oncoprotein, the curved red line represents miR-20b, the green oval represents TIMP-2 protein, and the irregular pattern represents cervica cancer cells. Arrows represent promotion, inverted $\mathrm{T}$ stands for inhibition. expression of MMP-2 and TIMP-2 in cervical carcinoma (17): both MMP-2 and TIMP-2 mRNA levels in tumor cells were associated with advanced-stage cancer, with poor survival. Braicu et al showed that serum TIMP-2 level could have predictive value in monitoring cervical cancer in patients undergoing radiochemotherapy (18). Moreover, Cardeal et al found that HPV oncoproteins promoted the matrix metalloproteinase (MMP)/TIMP-2 imbalance, which may be involved in HPV-associated lesion outcome (19). However, the interaction of HPV, miR-20b and TIMP-2 has not been studied in cervical cancer progression.

Therefore, we selected TIMP- 2 regulated by HPV and related to cell invasion for follow-up study of downstream target genes. Luciferase reporter gene assay confirmed that the TIMP-2 activity was downregulated after co-transfection with miR-20b and TIMP-2, which suggested TIMP-2 as an miR-20b-directed target gene. MiR-20b can affect the biological function of cells by regulating TIMP-2. We next confirmed that TIMP-2 was involved in cell invasion regulated by miR-20b and HPV E6. Previously, miR-20a inhibited the proliferation, migration and invasion of cervical cancer cells by targeting ATG7 and TIMP-2 (20). In addition, miR-20b also exerts different biological effects via different downstream target genes. MiR-20b reduced the expression of vascular endothelial growth factor 
by targeting hypoxia-inducible factor 1 and signal transducer and activator of transcription 3 in breast cancer (21). MiR-20b regulates the expression of the proteinase-activated receptor-1 thrombin receptor, which is associated with the degree of cancer invasiveness in melanoma cells (22).

MiR-20b is a newly discovered regulatory molecule in cervical cancer, and here we revealed its role and mechanism in the cancer. In particular, we preliminarily verified the relation between miR-20b, TIMP-2 and HPV. However, how HPV precisely regulates the expression of miR-20b has not been described. The Rbfox family of RNA-binding proteins contains the same sequence motif as miR-20b precursors in the inverted-repeated terminal loops, thereby depressing nuclear processing (23). Whether HPV is involved in the synthesis of the miR-20b precursor needs to be further explored.

In conclusion, we found that the HPV oncoprotein increases the miR-20b transcription in cervical cancer cells and degradation of TIMP-2, promotes cell invasion in cervical cancer (Fig. 6). In other words, miR-20b regulated by HPVE6 promotes cervical cancer invasion by targeting TIMP-2. This study revealed the important role of the HPV/miR-20b/TIMP-2 signaling pathway in cervical cancer.

\section{Acknowledgments}

The present study was supported by grants from the National Natural Science Foundation of China (grant no. 81472429), National Basic Research Program of China (973 Program, 2013CB933702), National Key Technology Research and Development Program of the Ministry of Science and Technology of China (2015BAI13B06).

\section{References}

1. Pisani P, Bray F and Parkin DM: Estimates of the worldwide prevalence of cancer for 25 sites in the adult population. Int $\mathbf{J}$ Cancer 97: 72-81, 2002.

2. zur Hausen H: Papillomavirus infections: A major cause of human cancers. Biochem Biophys Acta 1288: F55-F78, 1996.

3. Mendes de Oliveira $C$ and Levi JE: The biological impact of genomic diversity in cervical cancer development. Acta Cytol 60: 513-517, 2016.

4. Ambros V and Lee RC: Identification of microRNAs and other tiny noncoding RNAs by cDNA cloning. Methods Mol Biol 265: $131-158,2004$

5. Chiantore MV, Mangino G, Iuliano M, Zangrillo MS, De Lillis I, Vaccari G, Accardi R, Tommasino M, Columba Cabezas S, Federico M, et al: Human papillomavirus E6 and E7 oncoproteins affect the expression of cancer-related microRNAs: Additional evidence in HPV-induced tumorigenesis. J Cancer Res Clin Oncol 142: 1751-1763, 2016.

6. Ma D, Zhang YY, Guo YL, Li ZJ and Geng L: Profiling of microRNA-mRNA reveals roles of microRNAs in cervical cancer. Chin Med J (Engl) 125: 4270-4276, 2017.

7. Leidinger P, Brefort T, Backes C, Krapp M, Galata V, Beier M, Kohlhaas J, Huwer H, Meese E and Keller A: High-throughput qRT-PCR validation of blood microRNAs in non-small cell lung cancer. Oncotarget 7: 4611-4623, 2016.
8. Danza K, Silvestris N, Simone G, Signorile M, Saragoni L, Brunetti O, Monti M, Mazzotta A, De Summa S, Mangia A and Tommasi S: Role of miR-27a, miR-181a and miR-20b in gastric cancer hypoxia-induced chemoresistance. Cancer Biol Ther 17: 400-406, 2016.

9. Borges NM, do Vale Elias M, Fook-Alves VL, Andrade TA, de Conti ML, Macedo MP, Begnami MD, Campos AH, Etto LY, Bortoluzzo AB, et al: Angiomirs expression profiling in diffuse large B-Cell lymphoma. Oncotarget 7: 4806-4816, 2016.

10. Nieto MA: The ins and outs of the epithelial to mesenchymal transition in health and disease. Annu Rev Cell Dev Biol 27: 347-376, 2011.

11. Lan K, Zhao Y, Fan Y, Ma B, Yang S, Liu Q, Linghu H and Wang H: Sulfiredoxin may promote cervical cancer metastasis via Wnt/ $\beta$-catenin signaling pathway. Int J Mol Sci 18: pii: E917, 2017.

12. Hui AB, Lin A, Xu W, Waldron L, Perez-Ordonez B, Weinreb I, Shi W, Bruce J, Huang SH, O'Sullivan B, et al: Potentially prognostic miRNAs in HPV-associated oropharyngeal carcinoma. Clin Cancer Res 19: 2154-2162, 2013.

13. Wang X, Wang HK, Li Y, Hafner M, Banerjee NS, Tang S, Briskin D, Meyers C, Chow LT, Xie X, et al: microRNAs are biomarkers of oncogenic human papillomavirus infections. Proc Natl Acad Sci USA 111: 4262-4267, 2014.

14. Liu F, Zhang S, Zhao Z, Mao X, Huang J, Wu Z, Zheng L and Wang Q: MicroRNA-27b up-regulated by human papillomavirus $16 \mathrm{E} 7$ promotes proliferation and suppresses apoptosis by targeting polo-like kinase 2 in cervical cancer. Oncotarget 7: 19666-19679, 2016.

15. Acloque H, Adams MS, Fishwick K, Bronner-Fraser M and Nieto MA: Epithelial-mesenchymal transitions: The importance of changing cell state in development and disease. J Clin Invest 119: 1438-1449, 2009.

16. Kalluri R and Weinberg RA: The basics of epithelial-mesenchymal transition. J Clin Invest 119: 1420-1428, 2009.

17. Davidson B, Goldberg I, Kopolovic J, Lerner-Geva L, Gotlieb WH, Ben-Baruch G and Reich R: MMP-2 and TIMP-2 expression correlates with poor prognosis in cervical carcinoma-a clinicopathologic study using immunohistochemistry and mRNA in situ hybridization. Gynecol Oncol 73: 372-382, 1999.

18. Braicu EI, Gasimli K, Richter R, Nassir M, Kümmel S, Blohmer JU, Yalcinkaya I, Chekerov R, Ignat I, Ionescu A, et al: Role of serum VEGFA, TIMP2, MMP2 and MMP9 in monitoring response to adjuvant radiochemotherapy in patients with primary cervical cancer-results of a companion protocol of the randomized NOGGO-AGO phase III clinical trial. Anticancer Res 34: 385-391, 2014

19. Cardeal LB, Boccardo E, Termini L, Rabachini T, Andreoli MA, di Loreto C, Longatto Filho A, Villa LL and Maria-Engler SS: HPV16 oncoproteins induce MMPs/RECK-TIMP-2 imbalance in primary keratinocytes: Possible implications in cervical carcinogenesis. PLoS One 7: e33585, 2012.

20. Zhao S, Yao D, Chen J, Ding N and Ren F: MiR-20a promotes cervical cancer proliferation and metastasis in vitro and in vivo. PLoS One 10: e0120905, 2015.

21. Cascio S, D'Andrea A, Ferla R, Surmacz E, Gulotta E, Amodeo V, Bazan V, Gebbia N and Russo A: miR-20b modulates VEGF expression by targeting HIF-1 alpha and STAT3 in MCF-7 breast cancer cells. J Cell Physiol 224: 242-249, 2010.

22. Saleiban A, Faxälv L, Claesson K, Jönsson JI and Osman A: miR-20b regulates expression of proteinase-activated receptor-1 (PAR-1) thrombin receptor in melanoma cells. Pigment Cell Melanoma Res 27: 431-441, 2014.

23. Chen Y, Zubovic L, Yang F, Godin K, Pavelitz T, Castellanos J, Macchi P and Varani G: Rbfox proteins regulate microRNA biogenesis by sequence-specific binding to their precursors and target downstream Dicer. Nucleic Acids Res 44: 4381-4395, 2016. 